\title{
Congenital Atresia of the Ostium of the Left Coronary Artery. Diagnostic Difficulty and Successful Surgical Revascularization in Two Patients
}

\author{
Fernando Amaral, Carla Tanamati, João A Granzotti, Jorge L Haddad, João Ronaldo A Leite, \\ Miguel Barbero-Marcial \\ Ribeirão Preto, SP - Brazil
}

\begin{abstract}
We report two cases of congenital atresia of the ostium of the left coronary artery. Case 1: a six-month-old infant presenting with serious cardiac insufficiency. A noninvasive diagnosis of dilated myocardiopathy was established and the clinical picture was pharmacologically compensated. When the patient was nine months of age, a hemodynamic study was performed that revealed congenital atresia of the ostium of the left coronary artery; the infant immediately underwent a successful anastomosis of the internal mammary artery with the left coronary artery. Case 2: an eleven-year-old asymptomatic boy with a history of heart murmur from the age of six months on, was refered for surgery with a diagnosis of anomalous origin of the left coronary artery from pulmonary trunk. A definitive diagnosis of atresia of the left coronary ostium was only established during surgery. Successful surgical revascularization with the left internal mammary artery, and left ventricular aneurysmectomy were performed.
\end{abstract}

Congenital anomalies of the coronary arteries, occurring in $1-2 \%$ of the population ${ }^{1}$, are classified as anomalies at the origin (abnormal exit from the pulmonary trunk of the left coronary artery), terminal (coronary fistulae) and distributive (one coronary artery only) ${ }^{2}$. Congenital atresia of the ostium of the left coronary artery is a rather rare occurrence that is not even mentioned in the above-cited literature. Classically, it consists of total ostial atresia of the left coronary artery, possibly in association with atresia of the trunk of this vessel. Clinically, during the first months of life such patients may present with cardiac insufficiency (infantile form) or a delayed (including adults) picture of coronary insufficiency (adult form). We report below two patients of different ages and clinical presentations, having

Hospital do Coração de Ribeirão Preto/Fundação Waldemar B. Pessoa, Hospital das Clínicas da Faculdade de Medicina de Ribeirão Preto, USP and Instituto do Coração do Hospital das Clínicas da Faculdade de Medicina de São Paulo, USP Mailing address: Fernando Amaral - AV. Independência, 1379 - 14025-390, Ribeirão Preto, SP, Brazil congenital atresia of the ostium of the left coronary artery of difficult diagnosis, who underwent successful surgical myocardial revascularization.

\section{Case reports}

Case 1 - A nine-month-old boy from Divinolândia, SP, with a clinical history of tiredness on sucking, accompanied by discrete diaphoresis from the first month of life on. At six months of age, after a case of pneumonia, a sudden worsening of his condition occurred accompanied by cardiac insufficiency. A noninvasive investigation diagnosed dilated myocardiopathy. The patient showed significant improvement following medication, receiving a discharge from the hospital with a prescription of digoxin, furosemide and captopril. At nine months of age, he was referred to clinical examination at the Heart Hospital of Ribeirão Preto and he was found to be in excellent general condition (weight: $10 \mathrm{~kg}$ ). On physical examination, he had a normal pulse and upon cardiac auscultation $\mathrm{a}++/ 6$ systolic murmur on the $2^{\text {nd }}-3^{\text {rd }}$ left intercostal space with normal heart sounds. Thoracic antero-posterior radiography showed significant cardiomegaly (fig. 1 upper side). The electrocardiogram showed unequivocal signs of anterolateral infarction (fig. $2 \mathrm{~A}$ ). In view of this consistent clinical evidence, an initial diagnosis of anomalous origin of the left coronary artery from the pulmonary atretik was made. Due to lack of evidence of an anomalous coronary artery on a previously performed echocardiogram, a diagnostic hemodynamic study was indicated. Depressed left ventricular function was observed during this procedure. The right coronary artery was dilated, and collateral circulation nourished the left coronary, which was atretic in its ostium. No shunting to the pulmonary artery was detected (fig. 3). Once the diagnosis of congenital atresia of the ostium of the left coronary artery had been established, the infant immediately underwent surgical treatment by revascularization of the left coronary artery with a graft from the internal thoracic artery. The postoperative period was uneventfull, and 11 months following surgery the patient remains asymptomatic, in good general condition and under the use of digoxin. Thoracic radiography showed a decrease in cardiac area 


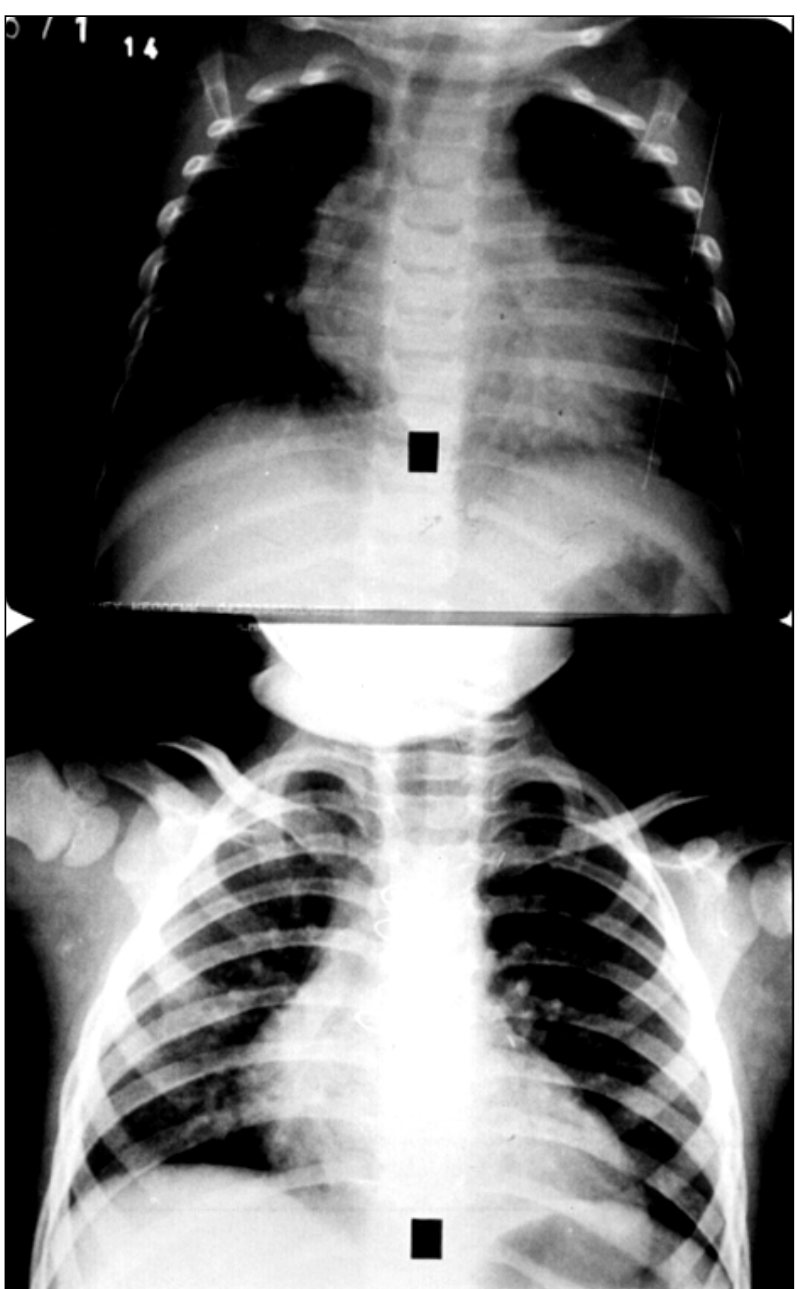

Fig. 1 - Antero-posterior thoracic radiography of case 1, showing preoperative cardiomegaly and signs of pulmonary congestion (upper); radiological improvement 11 months after surgery (lower).

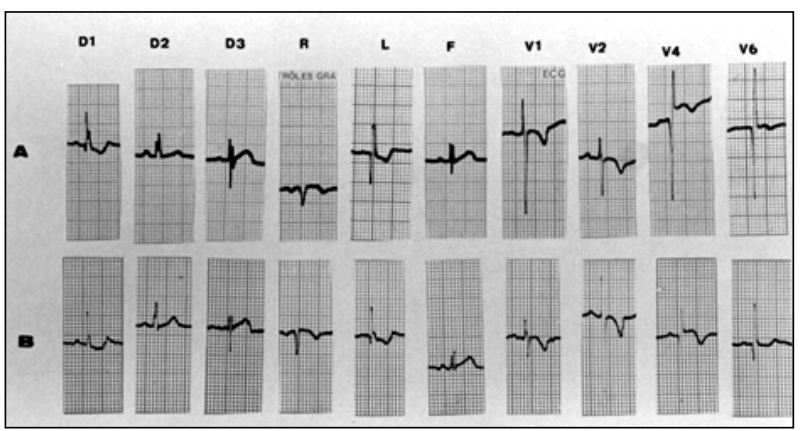

Fig. 2 - Electrocardiogram of case 1, showing preoperative signs of antero-lateral infarct (A) and improved ventricular repolarization of the anterior wall 11 months following surgery (B).

(fig. 1, lower side) and an improvement of electrocardiographic pattern (fig. 2B). A recent echocardiographic study still shows left ventricular dilatation and hypokinesia of the lateral wall, but also shows global improvement in the ejection fraction $(=0.72)$.

Case 2 - An eleven-year-old male from Franca, SP with a clinical history of cardiac murmur detected on a

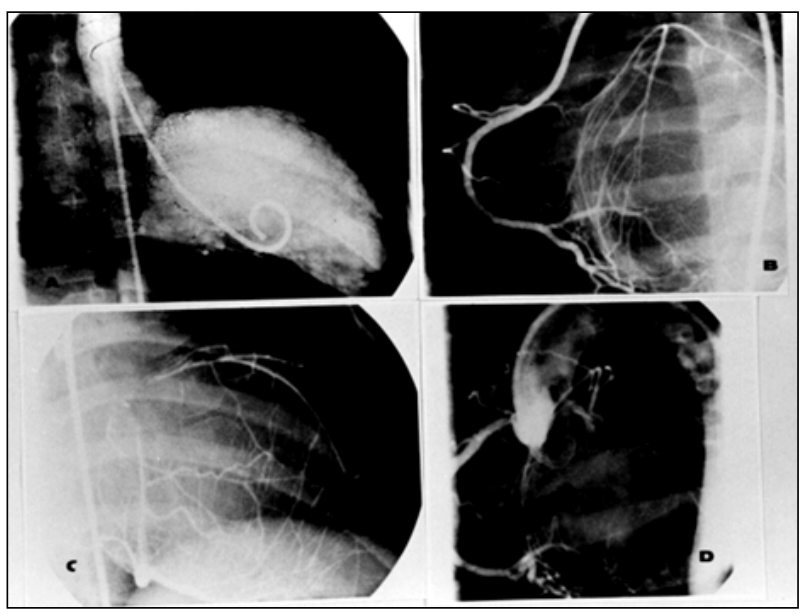

Fig. 3 - A) Preoperation hemodynamic study of case 1., showing left ventriculography in systole with marked diffuse hypokinesia: B) right coronariography in OLA showing dilated RCA with collateral circulation to LCA in the initial and final phase C) ; D) aortography of OLA showing normal opacity of RCA and contiguity but no communication of the LCA with the Valsalva sinus. OLA ostium of the left coronary artery; RCA - right coronary artery; LCA - left coronary artery.

routine examination when six months old was referred to the Heart Institute of São Paulo for diagnostic investigation and treatment. When the boy was $2 \frac{1}{2}$ years old, a hemodynamic study was performed that led to a diagnosis of anomalous origin of the left coronary artery from pulmonary trunk. Surgical treatment proposed at the time- was not accepted by the family; the child developed asthmatic bronchitis, no physical limitations and a good weight/structural growth pattern. Upon admittance to the hospital, he was observed to be in good general condition, had a palpable pulse and $++/ 6+$ ejection systolic murmur in the mitral area. Thoracic radiography showed a mild increase in the cardiac area; the electrocardiogram showed signs of left ventricular strain and an inactive high antero-lateral area (fig. 4 A). An effort test according to Bruce protocol detected upslope of the ST segment in $\mathrm{V}_{4}-\mathrm{V}_{6}$, compatible with antero-apical ischemia. The echocardiogram revealed an anomalous origin of the left coronary artery from the pulmonary trunk, slight mitral insufficiency and an apical aneurysm of the left ventricle with an ejection fraction of 0.69 . Thallium 201 scintigraphy during effort showed a persistent hypocaptation anteroseptal and apical regions; technetium-99m scintigraphy

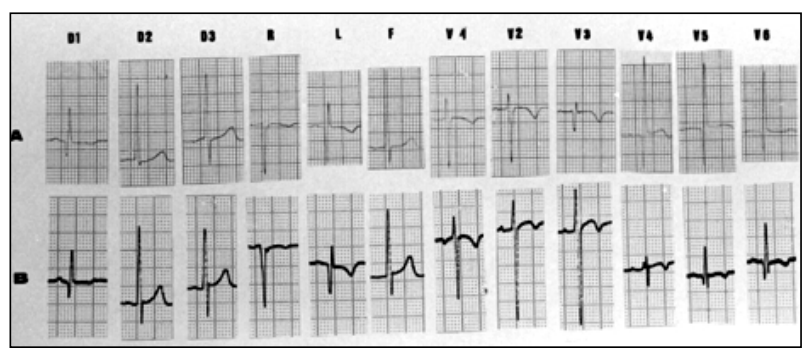

Fig. 4 - A) Preoperation electrocardiogram of case 2, showing deep q wave and alterations of ventricular repolarization on the antero-lateral wall; B) postoperation worsening of ventricular repolarization. 
revealed diffuse and moderate hypocontractibility of the left ventricle with an ejection fraction of 0.31 . A new hemodynamic study showed severe hypokinesia of the left ventricle with an antero-apical aneurysm and anomalous origin of the left coronary artery from the pulmonary trunk. Surgical myocardial revascularization and aneurysmectomy were indicated. Surgery revealed a large left ventricular aneurysm with marked diffuse hypokinesia. Under extracorporeal circulation, the pulmonary trunk was opened longitudinally, the left coronary ostium not becoming visible. The aorta was opened transversally; the impossibility of finding the left ostium suggested a diagnosis of atresia of the left coronary ostium. Using the left internal thoracic artery, myocardial revascularization of the left coronary artery followed by aneurysmectomy were performed. At present, 3 1/2 years following surgery, the youngster is asymptomatic and normal on physical examination. Thoracic radiography and the electrocardiogram denote signs of an anterolateral infarct with significant alterations of left ventricular repolarization (fig. 4 B).

\section{Discussion}

Left coronary ostium congenital atresia is an extremely rare disease; an incidence between $0.01-0.04 \%$ of "sole coronary" cases is admitted on the basis of results of necroscopy studies ${ }^{3}$. The rarity of this condition can be realized from its absence from a relevant, recently published study on congenital anomalies of coronary arteries ${ }^{2}$, and also from the fact that only 23 cases had been described before $1989^{4}$. In the last nine years, reports in the literature remain quite scarce, calling attention to the fact that only five cases were treated in a European center of reference, only one of a breast-feeding infant ${ }^{5}$; this experience has been recently amplified to include nine cases (Alain Serraf, personal communication).

The anatomical alteration consists of atresia of the ostium of the left coronary artery, which may be associated with hypoplasia or atresia of the trunk of this vessel. Occasionally, serious isolated stenosis of the ostium can be met with and it may have some importance concerning the surgical technique to be employed ${ }^{5}$.

From the clinical point of view, this anomaly may be classified as "infantile" (a precocious manifestation with cardiac insufficiency) or "adult" (manifestation of angina or sudden death); the role of the collateral circulation between the right and the left coronary arteries is considered vital at the moment of the clinical presentation of these patients. The diagnostic suspicion based on purely clinical data is difficult, because symptoms are nonspecific. On the other hand, complementary examinations play a fundamental role in the preliminary phase. Thoracic radiography may be normal in the "adult" type of the anomaly or exhibit cardiomegaly and signs of pulmonary congestion in infants with cardiac failure. The clinical picture may be easily mistaken for dilated myocardiopathy, as occurred in the first case here presented. The electrocardiogram may become a valuable diagnostic tool when a suspicion of this anomaly exists, as happened in the case of the first patient, where the electrocardiogram showed a pattern of antero-lateral infarction. An interesting phenomenon to remember is the resemblance of the clinical picture found in the patients with the anomalous origin of the left coronary artery of the pulmonary trunk ${ }^{6}$. This was our first diagnostic hypothesis in both cases, largely because of the electrocardiographic findings, as we have recently had the opportunity to demonstrate ${ }^{7}$. Theoretically, the diagnosis of atresia of the left coronary ostium may be obtained from the echocardiogram, in spite of difficulties due to individual anatomy. In this regard, the precise echocardiographic delimitation of the atresic portion may be difficult even if aided by Doppler. We believe this to have occurred in case 1. Diagnostic confirmation has been routinely made through the hemodynamic study, differential diagnosis of anomalous origin of the left coronary artery from the pulmonary trunk being of importance. In this regard, interruption of the retrograde flow in the trunk of the left coronary artery and the absence of opacity are worth noting.

In order to avoid the progress of the myocardial lesion and to control cardiac insufficiency in infants, surgery should be performed immediately after diagnosis. Despite the record of two unsuccessful cases in breast-feeding infants ${ }^{6,8}$, present experience shows that revascularization with the patient's own mammary artery is a viable procedure in these cases, some reports of operations of patients in the 3 to 11 year age range being available ${ }^{4,5}$. A technique of surgical angioplasty in children with obstructive anomalies of the coronary arteries, including left ostium atresia, has recently been described ${ }^{9}$. Although new, this procedure seems to be of interest for the preservation of material for future revascularization, because in some cases saphenous vein grafts were used. Another consideration to be taken into account at the moment of surgical indication is the prevention of sudden death. Older children, adolescents and even adults presenting with few symptoms should be operated on shortly after diagnosis in order to prevent such an outcome, as already reported ${ }^{10}$. Our patients reflect in a rather clear way, the clinical spectrum presented by the disease. In the first case the infant, after a period of serious cardiac insufficiency, was in excellent general condition, although having radiological and electrocardiographic signs of serious myocardial jeopardy. The surgical procedure was well tolerated, and based on the clinical condition and the improved results of the complementary examinations, the short-term postoperative state of the child may be considered rather satisfactory. The second case convincingly illustrates the clinical picture classified as the adult type by some authors; however, certain issues remain for discussion and should be pointed out. Initially, it should be noted that this patient was practically asymptomatic when resting prior to surgery and although having an obviously abnormal preoperative electrocardiogram showed no signs of ischemia, which was however, promptly evoked by the effort 
test and confirmed by radioisotope evidence. Another interesting occurrence worth mentioning because of its relevance to this case was the difficulty of a preoperative diagnosis in the face of the results of the hemodynamic study, which revealed an anomalous origin of the left coronary artery of the pulmonary trunk. In addition to these two findings, another one in particular deserves attention in this patient. Four years following surgery, electrocardiographic evidence of worsening secondary to segmental ischemia of the left ventricle, confirmed by radioisotope evidence, could be detected. Yet, in spite of this ischemic alteration, the patient remains fully asymptomatic following great effort. It is likely that a compensatory mechanism effectively regulates the myocardial oxygen supply and demand relationship, thus avoiding manifestations of angina.

Due to the small amount of experience available in this field, long-term prognosis in these two cases remains uncertain. It becomes clear however that a delay in the diagnosis and consequently of treatment of the patient must influence recovery of left ventricular function in a negative manner.

To conclude, congenital atresia of the left coronary artery's ostium is a rare disease. It should be considered when diagnoses of dilated myocadiopathy or anomalous origin of the left coronary artery from the pulmonary trunk in infants or in adults suspected of having coronary insufficiency are made. Although difficult, diagnosis may be obtained by echocardiography that for the time being at least is to be routinely confirmed by the hemodynamic study. Myocardial revascularization surgery with grafting of the internal thoracic artery seems presently to be the method of choice; however, the possibility of surgical angioplasty should be considered. The rarity of this lesion renders its casuistry uniformly small in different clinics, making its therapeutic standardization difficult. Nevertheless, short-term results are encouraging and long-term follow-up becomes important for documentation of the real benefits of the surgical methods for the improvement of myocardial function.

\section{References}

1. Engel HJ, Torres C, Page L Jr. Major variations in anatomical origin of the coronary arteries. Angiographic observations in 4.250 patients without associated congenital heart disease. Cathet Cardiovasc Diagn 1975; 1: 157-69.

2. Fernandes ED, Kadivar H, Hallman GL, Reul GJ, Ott DA, Cooley DA. Congenital malformations of the coronary arteries: the Texas Heart Institute experience. Ann Thorac Surg 1992; 54: 732-40.

3. Sharbaugh AH, White RS. Single coronary artery. JAMA 1974; 230: 243-6.

4. KohE, Nakagawa M, Hamaoka K, Sawada T, Oga K. Congenital atresia of the leftcoronary ostium: diagnosis and surgical treatment. Pediatr Cardiol 1989; 10: 159-62.

5. Serraf A, Baron O, Nottin E, at al. Atrésie ou sténose congénitale de l'ostium coronaire gauche. Revascularisation myocardique chez 5 enfants. Arch Mal Coeur 1993; 86: 587-91
6. Byrum CJ, Blackman MS, Schneider B, Sondheimer HM, Kavey REW.Congenital atresia of the left coronary ostium and hypoplasia of the left main coronary artery. Am Heart J 1980; 99: 354-8.

7. Amaral F, Carvalho JS, Granzotti JA, Shinebourne EA. Origem anômala da artéria coronária esquerda do tronco pulmonar. Perfil clínico e resultados a médio prazo do tratamento cirúrgico. Arq Bras Cardiol 1999; 72: 307-13.

8. Harada K, Ito T, Suzuki Y, Shiota T, Shimada K, Takada G. Congenital atresia of the left coronary ostium. Letter. Eur J Ped 1993; 152: 539-40.

9. Bonnet D, Bonhoeffer P, Sidi D, et al. Surgical angioplasty of the main coronary arteries in children. J Thorac Cardiovasc Surg 1999; 117: 352-7.

10. Hauwaert LGV, Dumoulin M, Moerman P. Congenital atresia of left coronary ostium. Br Heart J 1982; 48: 298-300. 